\title{
ESTUDO DA DINÂMICA DE PARTÍCULAS NÃO-ESFÉRICAS EM TAMBOR ROTATÓRIO VIA TÉCNICAS DE CFD
}

\author{
W. M. BENEDITO ${ }^{1}$, D. A. SANTOS ${ }^{1}$, C. R. DUARTE ${ }^{2}$ e M. A. S. BARROZO \\ ${ }^{1}$ Universidade Federal de Goiás, Instituto de Química \\ ${ }^{2}$ Universidade Federal de Uberlândia, Faculdade de Engenharia Química \\ E-mail para contato: mend.wanessa@gmail.com
}

\begin{abstract}
RESUMO - No presente trabalho foi realizado um estudo do comportamento dinâmico de partículas não-esféricas (grãos de arroz) no interior de um tambor rotatório por meio de técnicas de Fluidodinâmica Computacional (CFD). Os resultados obtidos de perfis radiais de velocidade de partículas via Modelagem Euleriana Granular Multifásica, foram comparados, sob as mesmas condições operacionais, com dados experimentais e com resultados numéricos via Modelagem Lagrangeana da literatura. Para as simulações numéricas aqui desenvolvidas, foram investigadas diferentes condições de contorno na parede, modelos de viscosidade granular friccional e coeficientes de restituição. Foi investigado, também, o efeito de parede sobre a dinâmica das partículas através da variação do comprimento do tambor. Os resultados preliminares se mostraram satisfatórios na utilização da abordagem Euleriana na modelagem de partículas não-esféricas no interior de tambores rotatórios. Porém, uma investigação mais detalhada acerca dos diferentes modelos de viscosidade granular deve ser realizada.
\end{abstract}

\section{INTRODUÇÃO}

O estudo da dinâmica de partículas no interior de tambores rotatórios tem se tornado cada vez mais importante em virtude da sua aplicação em diversos tipos de operações industriais como mistura (Gui et al., 2013), secagem (Scherer et al., 2016), dentre outras. A extensa empregabilidade de tambores rotatórios na indústria se deve ao fato de que estes são equipamentos com design simples, capazes de operar com partículas em uma ampla faixa de distribuição granulométrica e com diferenças significativas em suas propriedades físicas.

Os regimes de escoamento no interior de tambores rotatórios podem ser classificados, segundo Mellmann (2001), em: rolamento, cascateamento, catarateamento e centrifugação. Esta classificação depende diretamente das condições operacionais do equipamento, como velocidade de rotação $(\omega)$ e grau de preenchimento $(f)$, e das características do material particulado. No regime de rolamento, a superfície do leito de partículas se mantém plana, com ângulo de repouso dinâmico constante. É possível notar também neste tipo de regime a formação de duas regiões: uma região passiva, mais próxima à parede do tambor, na qual as partículas se movimentam com uma velocidade que se aproxima de um comportamento linear, e uma região ativa, próxima à superfície do leito de partículas, em que são encontrados maiores valores de velocidade e onde geralmente ocorrem transferências de massa e energia. 
Entre as regiões ativa e passiva, há um ponto de inflexão no perfil radial de velocidade de partículas, o qual caracteriza a mudança no sentido do escoamento granular. Faz-se importante, do ponto de vista do controle e da otimização de processos, a determinação das diferentes regiões formadas durante a operação do tambor rotatório por meio da distribuição de velocidades de partículas (Santos et al., 2015).

Devido ao grande avanço na área computacional, diferentes metodologias numéricas têm sido propostas com o intuito de sanar os problemas relacionados a custo de matéria prima, de confecção de equipamentos e de tempo dispendido na análise de processos complexos. As duas principais abordagens para o tratamento do escoamento granular são a aproximação Euleriana (CFD) e a aproximação Lagrangeana (DEM). Muitos autores já aplicaram estas duas abordagens numéricas no tratamento do escoamento granular para partículas esféricas em tambores rotatórios, obtendo resultados satisfatórios (Komossa et. al, 2014; Santos et al., 2015).

Como a maior parte dos materiais granulares utilizados industrialmente não possuem formato esférico, o presente trabalho visa o estudo do escoamento granular de partículas nãoesféricas em um tambor rotatório por meio da aplicação da metodologia Euleriana (CFD), utilizada na literatura quase que exclusivamente para partículas esféricas, e posterior comparação com dados experimentais e resultados simulados via metodologia Lagrangeana (DEM) advindos da literatura. Este trabalho também visa analisar o efeito de parede sobre a dinâmica das partículas no interior do tambor.

\section{MATERIAIS E METODOLOGIA}

\subsection{Metodologia Experimental}

Para a posterior comparação com os resultados aqui simulados, foram utilizados os dados experimentais obtidos por Santos et al. (2016) os quais utilizaram um tambor rotatório em escala de bancada, confeccionado em acrílico transparente, tendo 0,39 $\mathrm{m}$ de diâmetro, e dois diferentes comprimentos (L): $0,45 \mathrm{~m}$ e $0,02 \mathrm{~m}$. Como material particulado foram utilizados grãos de arroz com massa específica e diâmetro equivalente de $1465 \mathrm{~kg} / \mathrm{m}^{3}$ e 3,44 mm, respectivamente. O valor de porosidade do leito para os grãos de arroz foi de 0,47 .

A velocidade de rotação $(\omega)$ foi de $6,2 \mathrm{rpm}$ e grau de preenchimento do tambor $(f)$ de $10 \%$ (em volume) (regime de rolamento). Uma câmera de alta velocidade foi utilizada para obtenção dos perfis radiais de velocidade das partículas, tendo-se como base uma linha de referência localizada na metade da corda da superfície do leito de material.

\subsection{Metodologia Numérica}

Para as simulações numéricas desenvolvidas no presente trabalho foi empregada a Modelagem Euleriana Granular Multifásica juntamente com a teoria cinética do escoamento granular. Esta modelagem teve de ser readequada às partículas granulares não- esféricas, o que exigiu a avaliação de diferentes condições de contorno na parede, modelos de viscosidade granular e coeficiente de restituição. A modelagem Euleriana aplicada a sistemas particulados é descrita em detalhes por Santos et al. (2015).

Com relação às condições de contorno nas paredes laterais do tambor, foram analisadas as condições de não-deslizamento (no-slip) e as condições propostas por Johnson e Jackson 
(1987) com dois diferentes coeficientes de especularidade, sendo eles, 0 (o qual deveria corresponder, teoricamente, a uma condição de não-deslizamento) e 1 (deslizamento total). Foi verificado, também, o efeito da não-utilização de um modelo para o cálculo da viscosidade friccional, e a utilização do modelo proposto por Schaeffer (1987) com dois diferentes valores de fração crítica friccional de sólidos $\left(\alpha_{c r i ́ t i c o}\right): 0,41$ e 0,5 . Já para os coeficientes de restituição $\left(e_{s s}\right)$, foram analisados os valores de $0,1,0,6$ e 0,9 .

Para todos os cálculos numéricos, o resolvedor numérico Fluent ${ }^{\circledR}$ (versão 14.0) foi utilizado. A malha computacional empregada nas simulações foi confeccionada por meio da utilização do software Gambit. No intuito de comparar diferentes metodologias numéricas, foram utilizados os resultados numéricos obtidos por Santos et al. (2016) os quais empregaram a metodologia Lagrangeana através do software comercial PFC3D ${ }^{\odot}$ (Particle Flow Code 3-Dimensions).

\section{RESULTADOS E DISCUSSÕES}

\subsection{Investigação da Condição de Contorno na Parede, Viscosidade Granular e Coeficiente de Restituição na Modelagem Euleriana}

Em um primeiro momento foram realizadas diversas simulações no intuito de avaliar os efeitos decorrentes de diferentes condições de contorno nas paredes laterais do tambor. Foram selecionadas para esta avaliação as condições de não-deslizamento (no-slip) e as condições propostas por Johnson e Jackson (1987) com dois diferentes coeficientes de especularidade: 0 e 1. A Figura 1 mostra as distribuições de frações volumétricas de sólidos, experimentais e numéricas, no plano transversal do tambor de $0,45 \mathrm{~m}$ de comprimento (L).

Figura 1 - Efeito da condição de contorno $\left(f=10 \%, \omega=0,65 \mathrm{rad} / \mathrm{s}, \mathrm{L}=0,45 \mathrm{~m}\right.$; fixados $\alpha_{\text {crítico }}$ $=0,41$ e $e_{s s}=0,35$ ) - (a) experimental (Santos et al., 2016); (b) simulado CFD - no-slip; (c)

simulado CFD - especularidade 0; (d) simulado CFD - especularidade 1(0,45 m).
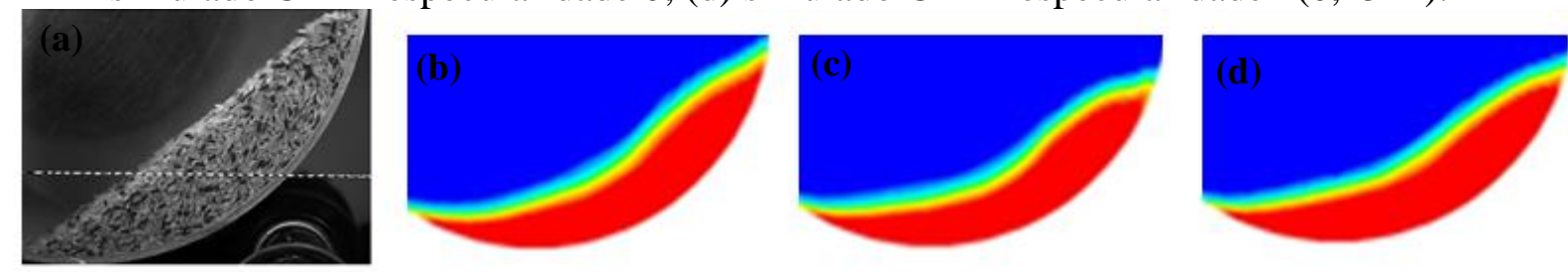

Através de uma análise qualitativa, foi possível concluir que, a configuração que mais se aproximou do perfil experimental foi aquela que utilizou uma condição de no-slip (Figura 1b), registrando um dos maiores ângulos de repouso para o leito de partículas, porém a superfície apresentou certa curvatura, o que não condiz com o regime de rolamento apresentado no experimental. Observa-se, também que, ao contrário do relatado na literatura, a condição de especularidade igual a 0 (Figura 1c) não condiz necessariamente a uma condição de não-deslizamento (no-slip) (Figura 1b). Logo, maiores atenções devem ser dadas no tratamento das condições de contorno na parede.

Em sequência, efetuou-se uma avaliação dos efeitos da viscosidade granular friccional sobre a dinâmica das partículas. Foi fixada nesta análise a condiçãode contorno de no-slip (melhor condição atestada anteriormente). Para tanto, realizou-se simulações sem levar em conta a utilização do termo de viscosidade friccional, e a utilização do modelo proposto por 
Schaeffer (1987) com dois diferentes valores de fração crítica friccional de sólidos $\left(\alpha_{c r i ́ t i c o}\right)$ : 0,41 e 0,5 . O valor de $\alpha_{\text {crítico }}=0,41$ foi mostrado anteriormente através da figura $1 \mathrm{~b}$, o que levou a uma superfície curva do leito de material. Por outro lado, a Figura 2 apresenta as distribuições de frações volumétricas de sólidos simulados sem modelo friccional e para $\alpha_{\text {crítico }}=0,5$.

Figura 2 - Efeito do modelo de viscosidade friccional $(f=10 \%, \omega=0,65 \mathrm{rad} / \mathrm{s}, \mathrm{L}=0,45 \mathrm{~m}$; fixados condição de contorno no-slip e $\left.e_{s s}=0,35\right)$ - (a) experimental (Santos et al., 2016) (b) simulado CFD - sem modelo friccional; (c) simulado CFD $-\alpha_{\text {crítico }}=0,5$.
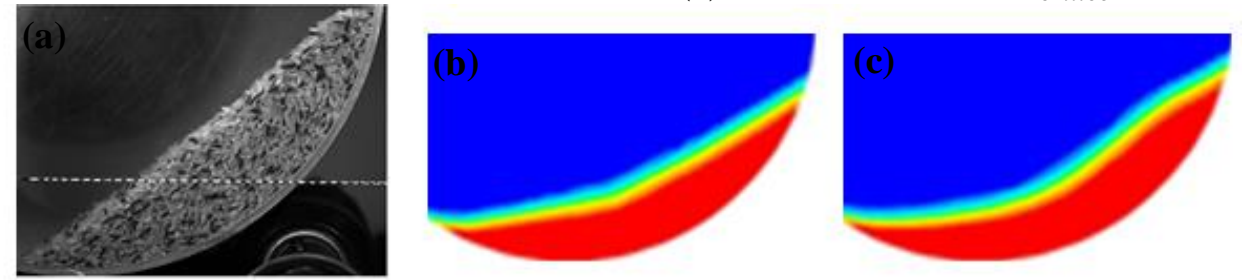

Nota-se através da Figura 2, que a configuração do modelo que não levou em consideração o efeito da viscosidade friccional (Figura 2b) melhor representou o regime de rolamento, ou seja, uma superfície mais plana. Diante dos resultados obtidos, investigou-se o efeito do coeficiente de restituição sobre as simulações, fixando-se agora, a condição de contorno de no-slip e desprezando-se os efeitos da viscosidade friccional. Foram utilizados três valores de coeficientes de restituição: $0,1,0,6$ e 0,9 , cujas distribuições de fração de sólidos simuladas são apresentadas na Figura 3.

Figura 3 - Efeito do coeficiente de restituição $(f=10 \%, \omega=0,65 \mathrm{rad} / \mathrm{s}, \mathrm{L}=0,45 \mathrm{~m}$; fixados condição de contorno no-slip e sem modelo friccional) - (a) experimental (Santos et al., 2016)

(b) simulado CFD $-e_{s s}=0,1$; (c) simulado CFD $-e_{s s}=0,6$; (d) simulado CFD $-e_{s s}=0,9$.
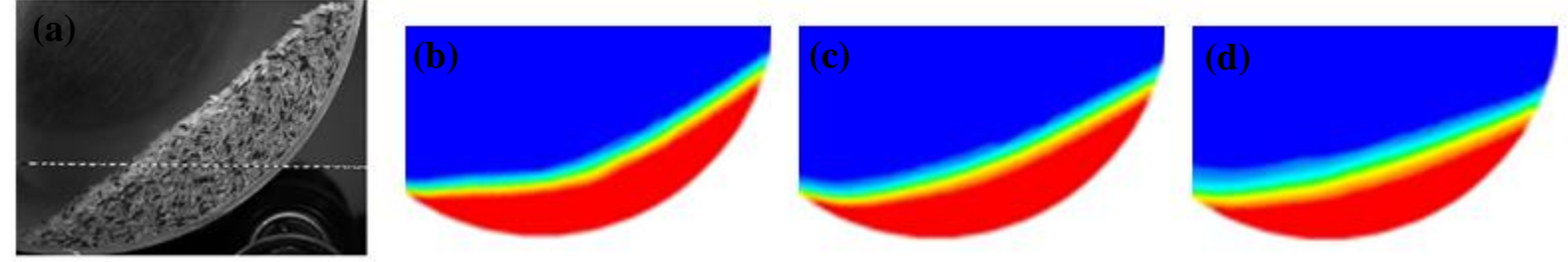

Nota-se que, a configuração que empregou $e_{s s}=0,6$ (Figura 3c), valor este condizente com os valores reportados na literatura para grão de arroz (Santos et al., 2016), foi a que ofereceu um perfil mais condizente com o regime de rolamento (superfície do leito de partículas praticamente plano), apesar da inclinação se apresentar menor do que no experimental. Desta forma, uma investigação mais detalhada acerca dos diferentes modelos de viscosidade cinético-colisional, dentre outras propriedades, deve ser feita para que o modelo Euleriano se adeque tanto à inclinação quanto à superfície plana de partículas não-esféricas escoando em regime de rolamento em um tambor rotatório.

Finalmente, a fim de se investigar o efeito de parede sobre a dinâmica das partículas no interior do tambor, a mesma configuração da Figura 3c foi aplicada em um tambor rotatório com um comprimento (L) de 0,02 m, cujas distribuições de sólidos experimental e simulado são apresentados na Figura 4. 
Figura 4 - Efeito de parede $(f=10 \%, \omega=0,65 \mathrm{rad} / \mathrm{s}, \mathrm{L}=0,02 \mathrm{~m})$ : (a) experimental (Santos $e t$ al., 2016); (b) simulado CFD - configuração de modelos da Figura 3c.
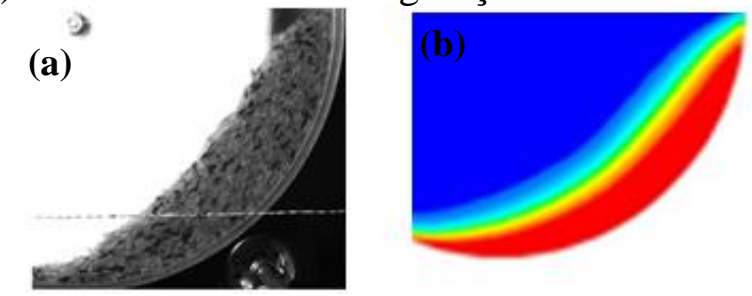

Pode-se observar que o perfil obtido pela simulação Euleriana (CFD) se mostrou semelhante ao perfil experimental. O leito de partículas ainda mantém-se no regime de rolamento, mas com certa iminência de transição para o regime de cascateamento (superfície do leito começa a curvar-se) e apresentando ângulo de repouso maior do que nas simulações com o tambor a $0,45 \mathrm{~m}$ de comprimento. Tais condições são diretamente decorrentes do efeito de parede ocasionado pela diminuição das dimensões do tambor.

\subsection{Investigação dos Perfis Radiais de Velocidade de Partículas Experimentais e Simulados}

Com o intuito de melhor avaliar as metodologias numéricas abordadas por este estudo e comparar os resultados fornecidos pelas simulações com os dados experimentais, perfis radiais de velocidade de sólidos utilizando dois diferentes comprimentos do tambor $(2 \mathrm{~cm} \mathrm{e}$ $45 \mathrm{~cm}$ ) foram colocados em um gráfico, juntamente com perfis simulados via CFD (utilizando a configuração da Figura 3c) e os perfis obtidos pela simulação Lagrangeana (DEM) (Santos et al., 2016) (Figura 5).

Figura 5 - Perfis radiais de velocidade de partículas simulado e experimental $(f=10 \%, \omega=$ $0,65 \mathrm{rad} / \mathrm{s}, \mathrm{L}=0,45$ e $0,02 \mathrm{~m}$ ) - (a) velocidade resultante; (b) componente $\mathrm{x}$ da velocidade.
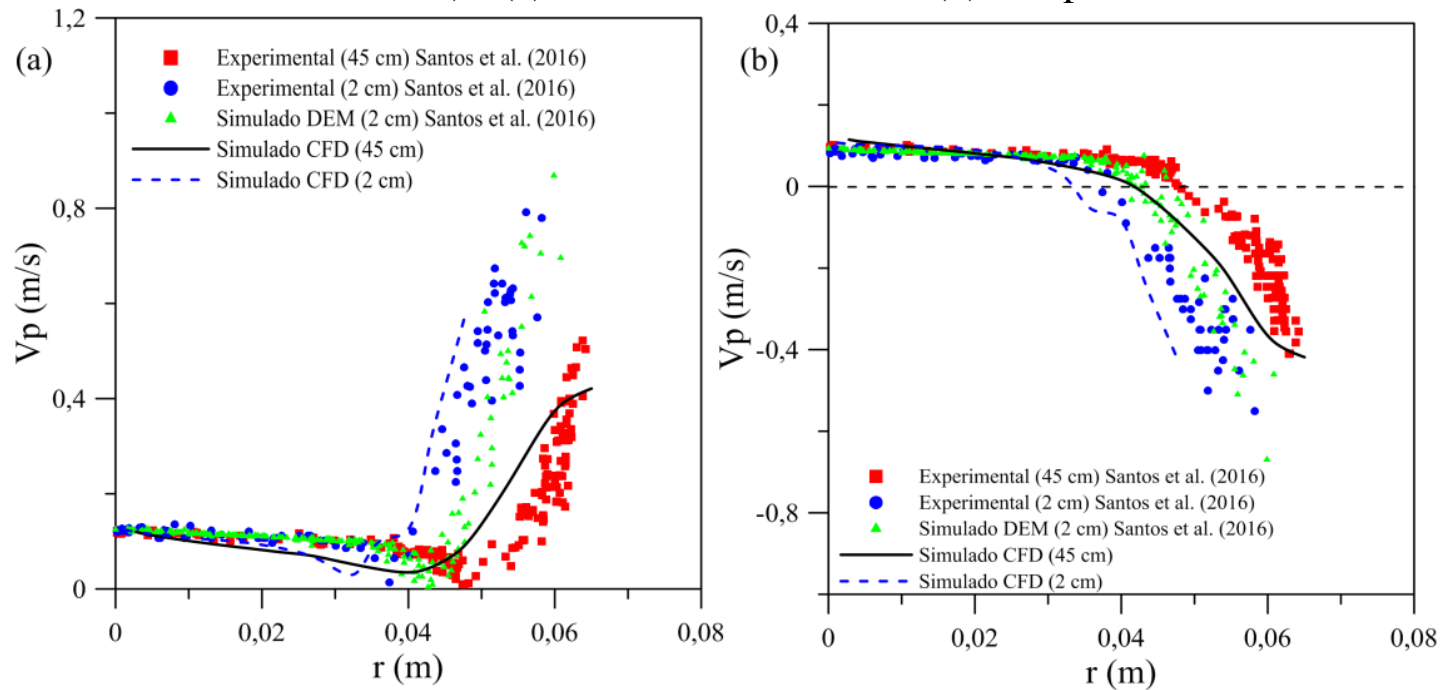

No intuito de se analisar o ponto de inflexão no perfil de velocidade de sólidos (ponto este onde a velocidade muda de sentido), foi colocado em um gráfico, além da velocidade de sólidos em magnitude (Figura 5a), a componente da velocidade de sólidos na direção x (Figura 5b). Observou-se que as propriedades do escoamento granular em um tambor rotatório, citadas anteriormente, como presença de região passiva, região ativa e ponto de inflexão, caracterizando a inversão do movimento de partículas na interface entre estas regiões, foram captadas por meio das simulações realizadas. 
Nota-se que a variação de comprimento do tambor possui influência sobre a região ativa. Para o tambor com $2 \mathrm{~cm}$ de comprimento foram registradas velocidades mais altas na região ativa quando comparado aos valores de velocidade alcançados nesta região para $\mathrm{o}$ tambor com $45 \mathrm{~cm}$ de comprimento. A diminuição do comprimento do tambor rotatório ocasionou um aumento do ângulo de repouso do leito de partículas. Este aumento está relacionado diretamente a um incremento na energia potencial das partículas, a qual é posteriormente convertida em energia cinética por ação gravitacional, proporcionando aumento das velocidades (Santos et al., 2016).

Nota-se que, ao contrário das simulações CFD, não foram realizadas simulações DEM para o tambor contendo $45 \mathrm{~cm}$ de comprimento devido ao alto custo computacional envolvido neste tipo de abordagem (Santos et al., 2016). As simulações Eulerianas (CFD) se mostraram satisfatórias quando da simulação do tambor de $45 \mathrm{~cm}$, porém apresentando uma velocidade máxima de partículas razoavelmente inferior ao experimental (Figura 5a). Este fato pode estar relacionado à menor inclinação do leito de partículas obtido nas simulações, como discutido anteriormente. Este mesmo efeito pôde ser notado quando da simulação no tambor com menor diâmetro (Figura 5). Apesar da simulação Lagrangeana (DEM) ter melhor representado os perfis de velocidade de sólidos, pois, ao contrário da simulação Euleriana, leva em conta a forma da partícula, a abordagem DEM se restringe a equipamentos com excessiva redução geométrica.

\section{CONCLUSÕES}

Os resultados preliminares se mostraram satisfatórios na utilização da abordagem Euleriana (CFD) na modelagem de partículas não-esféricas no interior de tambores rotatórios. A configuração envolvendo a condição de contorno no slip, sem modelo friccional e com coeficiente de restituição igual a 0,6, melhor representou os dados experimentais. Porém, uma investigação mais detalhada acerca dos diferentes modelos de viscosidade granular cinéticocolisional, além de outras propriedades do escoamento granular, deve ser realizada.

\section{REFERENCIAS}

GUI, N.; YAN, J.; XU, W.; GE, L.; WU, D.; JI, Z.; GAO, J.; JIANG, S.; YANG, X., DEM simulation and analysis of particle mixing and heat conduction in a rotating drum. Chemical Engineering Science, v. 97, p. 225-234, 2013.

JOHNSON, P. C., JACKSON, R., Frictional-collisional constitutive relations for granular materials with application to plane shearing, J. Fluid Mech., v.176, p. 67-93, 1987.

KOMOSSA, H.; WIRTZ, S.; SCHERER, V.; HERZ, F.; SPECHT, E., Transversal bed motion in rotating drums using spherical particles: Comparison of experiments with DEM simulations. Powder Technology, v. 264, p. 96-104, 2014.

MELLMANN, J., The transverse motion of solids in rotating cylinders - forms of motion and transition behavior. Powder Technology, v. 118, p. 251-270, 2001.

SANTOS, D. A.; BARROZO, M. A. S.; DUARTE, C. R.; WEIGLER, F.; MELLMANN, J., Investigation of particle dynamics in a rotary drum by means of experiments and numerical simulations using DEM. Advanced Powder Technology, v. 27, p. 692-703, 2016.

SANTOS, D. A.; DADALTO, F. O.; SCATENA, R.; DUARTE, C. R.; BARROZO, M. A. S., A hydrodynamic analysis of a rotating drum operating in the rolling regime. Chemical Engineering Research \& Design, v. 94, p. 204-212, 2015.

SCHAEFFER, G., Instability in the evolution equations describing incompressible granular flow, J. Differential Equations, v.66, p. 19-50, 1987.

SCHERER, V.; MÖNNIGMANN, M., BERNER, M. O.; SUDBROCK, F., Coupled DEMCFD simulation of drying wood chips in a rotary drum - Baffle design and model reduction. Fuel, v. 184, p. 896-904, 2016. 